\title{
Une forme spontanée de figuier (Ficus carica L.), le nābūt
}

Diversité de nomenclature, d'usage et de pratiques locales au Nord du Maroc

A spontaneous form of the fig tree (Ficus carica L.), the nābūt: diversity of nomenclature, use and local practices in Northern Morocco

Younes Hmimsa, Yildiz Aumeeruddy-Thomas et Mohammed Ater

\section{(2) OpenEdition}

Journals

Édition électronique

URL : https://journals.openedition.org/ethnoecologie/3186

DOI : 10.4000/ethnoecologie.3186

ISSN : 2267-2419

Éditeur

Laboratoire Éco-anthropologie

Référence électronique

Younes Hmimsa, Yildiz Aumeeruddy-Thomas et Mohammed Ater, « Une forme spontanée de figuier

(Ficus carica L.), le nābūt », Revue d'ethnoécologie [En ligne], Supplément 1 | 2017, mis en ligne le 17 octobre 2017, consulté le 19 novembre 2021. URL : http://journals.openedition.org/ethnoecologie/ 3186 ; DOI : https://doi.org/10.4000/ethnoecologie.3186

Ce document a été généré automatiquement le 19 novembre 2021.

Revue d'ethnoécologie est mis à disposition selon les termes de la licence Creative Commons Attribution - Pas d'Utilisation Commerciale - Pas de Modification 4.0 International. 


\title{
Une forme spontanée de figuier (Ficus carica L.), le nābūt
}

Diversité de nomenclature, d'usage et de pratiques locales au Nord du Maroc

\author{
A spontaneous form of the fig tree (Ficus carica L.), the nābūt: diversity of \\ nomenclature, use and local practices in Northern Morocco
}

Younes Hmimsa, Yildiz Aumeeruddy-Thomas et Mohammed Ater

\section{Introduction}

1 Les plantes alimentaires sauvages suscitent un grand intérêt de la communauté scientifique partout dans le monde, en Europe (Luczaj et al. 2012, Tardio et al. 2006, Simkova \& Polesney 2015) ou ailleurs (Neviditha 2017, Lulekal et al. 2011). Cet intérêt concerne essentiellement deux champs disciplinaires, par ailleurs très proches. Tout d'abord, l'ethnobiologie qui s'intéresse à ces plantes sous des angles variés mais que nous pouvons ramener essentiellement à la connaissance et la conservation des savoirs et cultures locaux (Pardo-de-Santayana et al. 2007, Akpavi et al. 2013). L'autre champ important est celui du domaine des ressources génétiques (Galluzzi et al. 2010, McKey et al. 2012). Ce dernier aspect est actuellement mis en avant avec les problématiques de la conservation de la biodiversité en relation avec les changements globaux (CastañedaÁlvarez et al. 2016).

2 Parmi les plantes alimentaires sauvages, nous nous intéressons particulièrement, aux espèces fruitières. Bien que la diversité des espèces fruitières spontanées, c'est-à-dire poussant par multiplication des graines, sans l'aide des hommes, soit moins importante en zone méditerranéenne comparativement aux zones tropicales, les exemples ne manquent pas. Pour illustrer nos propos, nous allons nous limiter à des exemples marocains où plusieurs espèces fruitières sauvages sont bien connues aussi bien pour des usages alimentaires que médicinaux. 
3 D'une manière générale, nous pouvons distinguer chez les fruitiers spontanés deux types: un type qualifié de sauvage sans équivoque car non touché par la domestication et n'ayant de ce fait pas son double dans le domaine cultivé, et des types que nous qualifions de spontanés car ils dériveraient d'espèces domestiquées. Ces types sont aussi appelés dans la littérature "échappés de culture ». La distinction entre formes domestiquées d'une part et les formes spontanées et sauvages d'autre part, peut se faire chez les espèces pérennes et ligneuses par le mode de reproduction. Chez les formes domestiquées, la reproduction se fait surtout par multiplication végétative, à l'exception de l'amandier (Delplancke 2011) alors que pour les formes spontanées elle se fait plutôt par régénération par semis spontanés. Pour illustrer cette distinction on peut citer comme exemples de fruitiers sauvages bien connus au Maroc, l'arbousier (Arbutus unedo L.), le jujubier (Zizyphys lotus (L.) Lam.), les ronces (Rubus ulmifolius Shott) ou le caroubier ${ }^{1}$ (Ceratonia siliqua L.).

4 En ce qui concerne les fruitiers domestiqués, ils sont présents dans les agroécosystèmes traditionnels où persistent encore différentes formes de savoir traditionnels à l'exception de l'expansion industrielle de la culture de l'olivier à l'échelle nationale (Aumeeruddy-Thomas \& al. 2016). Dans les agroécosystèmes de type oasien on peut citer plusieurs exemples dont l'espèce clé de voute, le palmier dattier (Phoenix dactylifera). Parmi les variétés recensées, on reconnait les khalt nom générique regroupant les palmiers issus de semis naturel spontané (probablement des hybrides des variétés de dattiers dont les parents sont inconnus) et qui représentent plus de $55 \%$ de l'effectif total des palmiers (INRA 2011). Dans le même agroécosystème, on peut citer d'autres espèces comme celui bien étudié de l'abricotier (Prunus armeniaca L.) (Bourguiba et al. 2012, Mamouni et al. 2013) et celui moins bien connu du grenadier qui fait l'objet de travaux en cours. Pour les agroécosystèmes des montagnes rifaines plus représentatifs des régions méditerranéennes, la situation est plus équivoque, car il est plus difficile de distinguer les formes spontanées sauvages des formes d'origines domestiquées. En effet, si on écarte l'exemple de la vigne (Vitis vinefera ssp. sativa), où les formes sauvages (Vitis vinifera ssp. silvestris ou lambrusque) sont faciles à distinguer et si on prend l'exemple de l'olivier (Olea europea ssp. europea var. europea) on se rend compte que le statut des formes sauvages (Olea europea ssp europea var. sylvestris) n'est pas toujours facile à affirmer. En effet, la présence de formes ferrales ${ }^{2}$ (issues de semis) est attestée et elles sont utilisées par les paysans au même titre que celles provenant de la forêt, car pour ces derniers, il n'y a pas de ferrales d'une part et de sauvage de l'autre, sur le plan des pratiques et des savoirs locaux (Aumeeruddy-Thomas et al. 2017).

5 Dans ce contexte, on ne peut pas ignorer le figuier (Ficus carica L.) espèce emblématique des agroécosystèmes traditionnels du Rif (Hmimsa 2009, Aumeeruddy-Thomas 2010, Aumeeruddy-Thomas et al. 2014). En effet, dans nos travaux sur cette espèce nous avons relevé la présence de formes spontanées en coexistence avec les formes cultivées. Cependant, le statut de forme sauvage reste difficile à reconnaitre lors des prospections. En effet, d'une manière générale la distinction entre forme sauvage et domestiquée se fait à partir des caractères du syndrome de domestication. Or ces caractères ne sont pas bien développés chez les formes pérennes multipliées végétativement (Khadari et al. 2005, Tavaud et al. 2004). Ainsi, les manifestations apparentes du passage du spontané au cultivé se réduisent chez le figuier à la présence des fruits parthénocarpiques (figue fleur) (Khadari et al. 2005). Du point de vue pratique, dans les prospections, les généticiens se basent surtout sur les 
caractéristiques de l'habitat naturel (Khadari et al. 2005) pour différencier les formes spontanées qui seraient les plus proches des formes sauvages, à savoir:1) des sites naturels avec sols rocailleux, 2) situés sur des cours d'eau, 3) végétation ouverte sur des pentes fortes. Ces travaux ne tiennent cependant pas compte des distances de dispersion des graines de figuiers cultivés ou du cadre spatio-temporel de l'occupation historique des vergers à figuiers.

6 Au Maroc, l'arboriculture fruitière s'est développée dans différentes régions et s'est intégrée dans différents types d'agroécosystèmes. Actuellement, les arbres fruitiers représentent un élément structurant des agroécosystèmes marocains, tels que le palmier dattier pour les oasis, la coexistence d'une grande diversité d'espèces arborées (oliviers, amandiers, noyers, caroubiers etc.) pour les systèmes agro-sylvo-pastoraux de l'Atlas et l'arganier (Argania spinosa (L.) Skeels) pour les arganeraies. Elle est généralement de type extensif et concerne surtout des arbres de haute-tige, très espacés pour laisser la place à des cultures complémentaires, notamment des prairies ou des céréales. Parfois, elle peut faire partie d'une filière particulière et fournir des fruits destinés à l'industrie de la transformation.

Depuis des temps reculés, la richesse en arboriculture fruitière a caractérisé les agroécosystèmes des montagnes rifaines (Léon l'africain 1550, Focauld 1883-1884, Fay 1972, Tayou 1985). En effet, l'arboriculture occupait une place plus prépondérante par rapport aux céréales dans le passé pour la production de raisins secs et de figues séchées. Ces productions importantes étaient échangées contre les céréales en provenance de la plaine du Gharb (Tayou 1985). Ces cultures étaient tellement importantes qu'elles marquaient le paysage. En effet, plusieurs citations historiques dénomment les montagnes du Rif comme Jbal Zbib (montagnes des raisins secs) (Léon l'Africain 1550).

8 A l'échelle nationale, le figuier est considéré au Maroc comme une espèce fruitière à importance secondaire (Walali Loudyi 2002, Oukabli 2003). Cependant, il en est autrement à l'échelle régionale où il peut avoir une grande importance. C'est le cas des agroécosystèmes traditionnels du Rif, marqués par une grande richesse de dénomination locale représentant 133 types nommés (Hmimsa et al. 2012) couvrant une grande diversité génétique (Achtak et al. 2010). Comprendre la dynamique de cette diversité implique la prise en compte des pratiques techniques et sociales liées à la sélection et au maintien de la diversité. L'étude des savoirs locaux et des pratiques en relation avec la nature constitue un champ de recherche ayant pour objectif de mettre en lumière les rapports des sociétés à la nature et les relations des hommes entre eux à ce propos. Dans ce contexte, l'étude a été focalisée sur les agroécosystèmes traditionnels rifains car l'élément le plus représentatif de la composante fruitière de ces agroécosystèmes est le figuier. Tenant compte de la biologie du figuier, nous allons présenter la diversité du figuier telle qu'elle est perçue par les agriculteurs à travers l'exemple du type nommée nābūt. Ainsi, on se propose de comprendre les facteurs favorisant la dualité spontanée - sauvage / domestiqué - cultivé, dans les vergers traditionnels et d'étudier cette coexistence en fonction des espaces, des pratiques et des usages. 


\section{Aire d'étude}

9 L'aire d'étude correspond aux montagnes du Rif au nord du Maroc. Ainsi, nous avons prospecté 208 « douars ou dšar » (Figure 1, Tableau 1), dans lesquels nous avons mené des entretiens avec des agriculteurs.

Figure 1 : Carte de localisation des sites de prospection au nord du Maroc

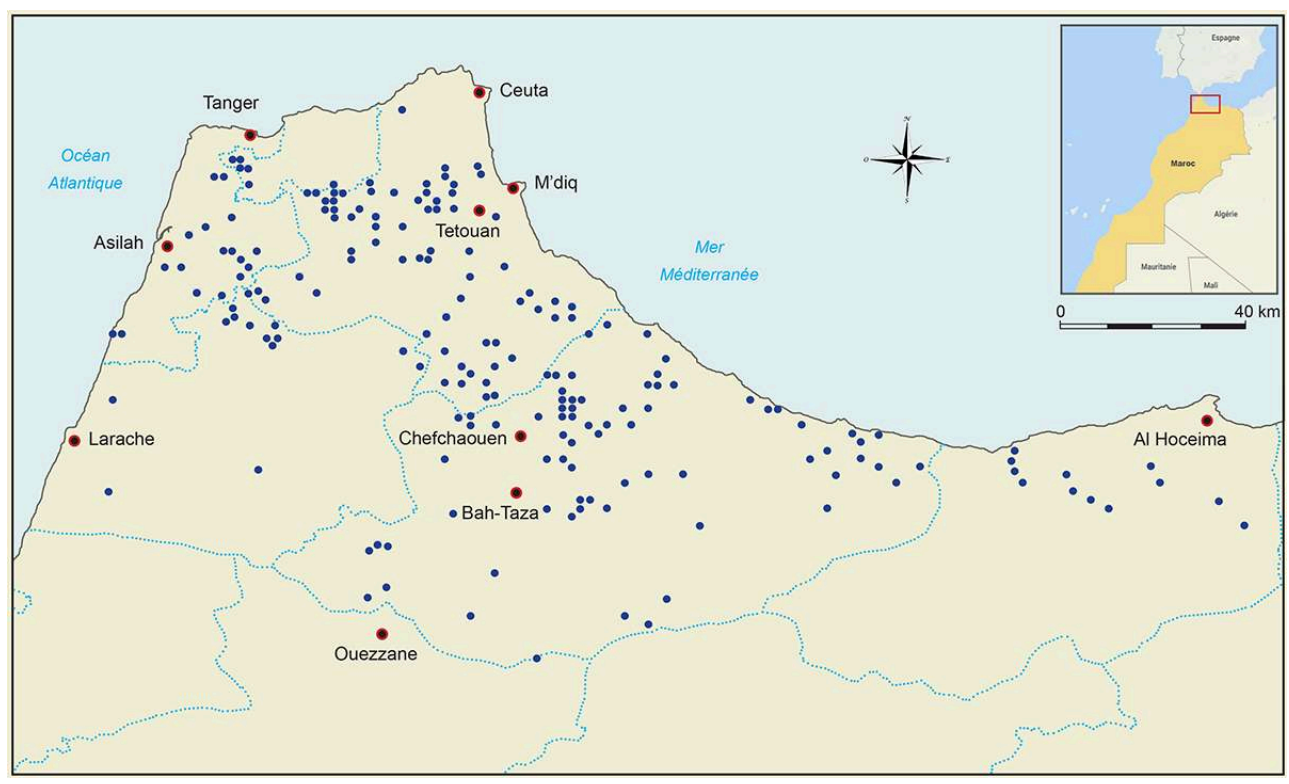

Du point de vue du cadre physique et géographique, il s'agit d'une unité intensément morcelée composée par la juxtaposition de compartiments contrastés sur le plan du relief, de la lithologie et de la structure (Maurer 1968, Lery 1982). La région est très humide (Benabid 1982), puisqu'elle reçoit les pluies les plus abondantes du pays (> $1000 \mathrm{~mm}$ ), quoiqu'on puisse observer une diminution des précipitations en direction du littoral méditerranéen et vers l'est (1,191 $\mathrm{mm}$ à Bab Berred, 1,542 $\mathrm{mm}$ à Ketama et $419 \mathrm{~mm}$ à Targuist) (Laouina 1998). Sur le plan sociodémographique, la population du Rif est constituée par une mosaïque de composantes qui a donné naissance à trois groupes ethniques principaux (Jbala, Ghomara et Zènète) (Maurer 1968, Teniente 1928, Mimoun 2003, Ait Kaki 2003). Du point de vue sociolinguistique, ils forment deux ensembles: arabophone dans la partie occidentale et berbérophone dans la partie orientale (Camps 1983, Moscoso 2003, Vicente et al. 2017). 
Tableau 1 : Liste des différents sites prospectés au cours de cette étude

\begin{tabular}{|c|c|}
\hline Province & Village \\
\hline $\begin{array}{l}\text { Al Hoceima } \\
\text { (10) }\end{array}$ & $\begin{array}{l}\text { Bni bousetta ; Boukora ; Torres de alcala ; Snada ; Imouraten ; Aamayer ; Tamasint ; Ait Aaziz ; Tadkant ; Tazaghine ; Jnanate ; } \\
\text { Bni Boufrah ; }\end{array}$ \\
\hline $\begin{array}{l}\text { Chefchaouen } \\
\text { (83) }\end{array}$ & 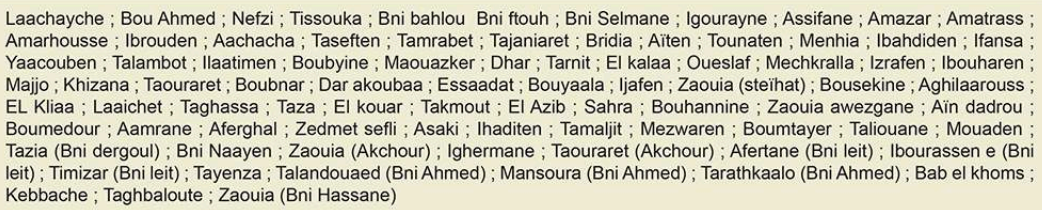 \\
\hline $\begin{array}{l}\text { Larache } \\
(22)\end{array}$ & $\begin{array}{l}\text { Elhsen ; Tazya ; Er rmel ; Al kharoub ; Dar Bensaddouk ; El khomss ; Dar el khayl ; Ain ksab ; Ain aabed ; Boukanour ; Zitouna ; } \\
\text { Khandak Hmar ; Ain Gazroul ; Abiat ; El brahma ; Tazemart ; Charkia ; El khaloua ; Boujediane ; Khmiss Sahel ; Laaoumara }\end{array}$ \\
\hline $\begin{array}{c}\text { Tanger } \\
(42)\end{array}$ & $\begin{array}{l}\text { Renouna ; Bni meslem ; Tafza ; Oulad el mehdi ; Oulad antar ; Aroua ; Laghmamda ; Badriouène ; Chouikrech ; Ain dalia kbira ; } \\
\text { Hajra en Nahl ; Ben saaid bou ammar ; Ben hjalat ; Ain el assel ; khendek ezarzour ; Ain dalia sghira ; Ain bellout ; Bougdour ; } \\
\text { Squedla ; Kahaoucha ; El har ; El hajra ; Dechra ahrek; Hamiouch ; Kanoua ; Bou hdifa ; Dar fellak ; Es Soua ; El mrah ; Rhdir } \\
\text { edefla ; Brarak; El mjebha ; Gharifa; ; Mlalhaine ; Jwamaa ; Edhar el oueste ; Ed daya ; El haoud ; El mkhaled ; Khandek el Had ; } \\
\text { Kdioua ; Jwaneb }\end{array}$ \\
\hline $\begin{array}{l}\text { Tétouan } \\
(44)\end{array}$ & $\begin{array}{l}\text { Adrou ; Tardan ; Kalaline ; Bettara ; Bni salem ; Bouzerlale ; El kouf fouki ; El bayen ; Menkoulou ; Dar el razi ; Dar el halka ; } \\
\text { Ahlou ; Al hamma; Iherassen ; Frane Ali ; Ihetachen ; Rdir el haj ; El onsra ; Zkaloua ; El aatnoune ; Abdelouich ; Ain moulatem } \\
\text { Oued el lile ; Chekrad ; Tafradet ; El onsar ; El gzira ; Bni saleh ; Oued khmiss; Choukhen ; Belwazene ; Oued Zarjoune ; El } \\
\text { aalaouiya ; Jibech ; El keddane ; Oued Agla ; Tarrant ; Agla ; Dhar Lahdida ; Ikhoujja ; Taghzout, Amtel, Toughza, laasfa }\end{array}$ \\
\hline $\begin{array}{l}\text { Ouezzane } \\
\text { (7) }\end{array}$ & Brikcha ; Hararra ; Agda ; Dwaher ; Ghwiba ; Ben ouella \\
\hline
\end{tabular}

\section{Méthodologie}

Dans cette étude, nous nous sommes intéressés exclusivement aux variétés locales nommées et reconnues par les paysans. Les variétés locales sont entendues dans le sens d'unités définies et gérées par les agriculteurs sur une zone géographique déterminée (Sadiki et al. 2002, Soriano Niebla 2004). En effet, les agriculteurs distinguent ces unités à partir de critères qualitatifs d'appréciation et des appellations propres associés à un ensemble de pratiques de taille, de pollinisation, de protection qui les rend très proches individuellement.

12 Cette démarche s'est appuyée sur une analyse comparative grâce à une étude entamée dans différents sites et à différentes échelles. Les données ont été obtenues à travers des enquêtes auprès des agriculteurs et des discussions semi directives couplées à des observations participantes sur le terrain. Nous avons focalisé notre attention sur la distinction entre les différents types de figuiers. La diversité des types d'arbres et/ou la diversité des statuts ont été analysés en fonction de la perception des agriculteurs, des espaces et des usages. Pour les analyses linguistiques nous allons essayer d'établir l'origine et le sens des noms vernaculaires récoltées.

\section{Résultats et discussions}

\section{Place du terme nābūt dans le langage de la culture du figuier}

Le nom vernaculaire de nābūt est un mot générique qui dérive du mot arabe classique ) نبتة nabta) nabate signifiant une plante. Du point de vue linguistique, la racine arabe $\mathrm{du}$ terme peut être originaire de $\{\mathrm{nb}$ ?\} pour faire référence à quelque «chose qui s'élève au-dessus du sol» (Corriente et al. 2017). Dans ce sens, le terme peut désigner aussi, un sauvageon, ou un arbre fruitier qui a poussé spontanément sans avoir été planté (Iraqui 1993). À ce stade, on peut considérer que le nābūt se fait soi-même, sans l'intervention de l'homme, autrement dit, il s'agit d'un arbre issu de semis (Sonnini et al. 1810). Ainsi, le terme fait allusion à des plantes spontanées d'origine naturelle. Le 
spontané, d'après le dictionnaire de la langue française (Larousse 2017), désigne un phénomène qui se produit sans avoir été provoqué, d'où en botanique, le terme « se dit des plantes qui croissent naturellement dans un pays sans y avoir été introduites par l'homme ».

14 Cette présentation du terme est en parfaite adéquation avec le sens que les agriculteurs, attribuent au terme de nābūt dans les agroécosystèmes traditionnels du Rif. En fait, le terme est généralisé pour « tout arbre qui a poussé tout seul à partir d'une graine qui a germé sans l'intervention humaine». Dans ce sens, les paysans considèrent souvent ce type d'arbre comme un don de dieu, en effet, il est perçu par la population comme étant issu d'un figuier spontané remontant à une époque que seul Dieu connaît. La population dans certains cas peut adopter, multiplier voir même utiliser ou consommer. Pour cela, en adoptant une telle pratique, on peut dire que le simple acte d'adopter une bouture d'un arbre issue d'un semis provenant de la grâce de Dieu, la multiplier et lui attribuer un nom, peut être perçu comme étant une forme de domestication.

15 Dans un autre contexte, le terme nābūt peut aussi être attribué à un type de figue séchée. En effet, et contrairement à la figue d'automne ou d'été qui sont cueillis et déposés dans une aire de séchage, le nābūt surtout dans la région de Bni Boufrah, désigne la figue d'automne qui est séchée sur les branches d'arbre de figuier. Ce même type de figue sèche est connu dans la région d'Ouezzane sous le nom de qāid ${ }^{4}$, dans un sens de qualité et pour faire allusion à l'autorité et le pouvoir qu'ont ces fruits qui restent accrochés à l'arbre et prennent leur temps pour sécher. Cette interprétation ne diffère pas de celle qu'on peut attribuer au terme de nābūt qui reflète un séchage qui ne nécessite pas l'intervention humaine.

\section{Diversité des types de figuier}

16 Le figuier est un arbre dioïque, dont le pied mâle et le pied femelle sont distincts. Les agriculteurs, reconnaissant la nature diö̈que de cet arbre, distinguent et nomment différemment les arbres selon leur sexe, la production, le lieu de développement... (Figure 2) 
Figure 2 : Interaction entre les deux compartiments cultivé - spontané

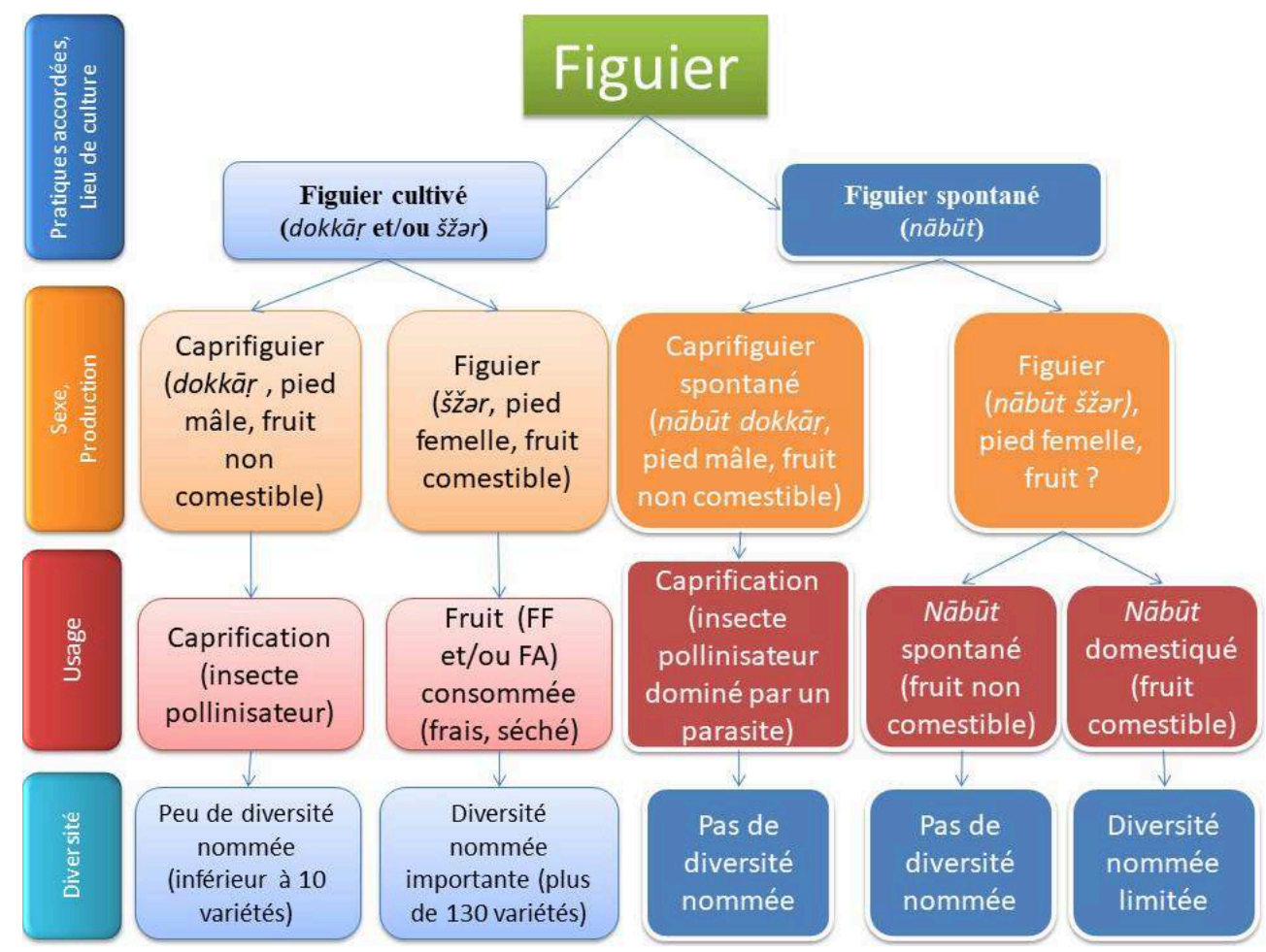

\section{Le caprifiguier}

17 Reconnu localement sous le nom du dokkār terme qui est utilisé par les paysans pour désigner le pied mâle du figuier et qui dérive du mot arabe marocain dkar ou $d$-dkar signifiant le sexe mâle/masculin. A ce niveau, on se trouve face à une distinction sexuelle permettant de différencier le figuier mâle du figuier femelle, dont, le lien entre le type de figuier et le sexe de l'arbre est une forme de représentation (Hmimsa et al. 2012, Hmimsa et al. 2017). Les critères biologiques adoptés pour classer les figuiers en mâle ou femelle dans la perception locale, sont les suivants :

- Le caprifiguier est le mâle parce qu'il ne produit pas de fruit comestible

- Le caprifiguier est le mâle qui est essentiel pour la production des fruits comestibles par le pied femelle.

De point de vue richesse variétale, à l'échelle du Rif, nous n'avons pas rencontré une grande diversité de types nommés de caprifiguiers, les agriculteurs regroupent sous le nom de $d k a r$ ou $d$-dkar l'ensemble de la diversité du caprifiguier. Cependant, chez les Bni Ahmed et dans la zone de Targuist (Figure 3), nous avons relevé des appellations correspondant à des variétés de caprifiguier qui sont respectivement au nombre de trois (hlu, marr et lwizi) chez les Bni Ahmed et de quatre (ahurri, aharchiw, ahfriw et azundri) au niveau de Targuist ${ }^{5}$. Au niveau des marchés hebdomadaires, les paysans distinguent entre les variétés et jugent leur qualité par l'abondance de l'insecte pollinisateur qui est le blastophage (Blastophaga psenes). Les profichis ou caprifigue (fruit du figuier mâle) sont récoltés et suspendus sur les branches des arbres de figuiers femelles afin de faciliter la pollinisation. Cette pratique se limite aux variétés de figuiers non parthénocarpiques. Une autre pratique concernant le caprifiguier consiste à transformer le caprifiguier en un figuier femelle. La transformation se fait par 
greffage, et le caprifiguier sert alors de porte greffe. Parfois, la transformation n'est pas complète, car nous avons observé des cas ou il existe sur le même pied une partie caprifiguier et une partie figuier. Du point de vue répartition dans l'espace, lorsqu'il est planté, on peut le rencontrer au milieu des vergers comme dans les bordures.

Figure 3 : Marché de caprifigues à Bni Ahmed (A) et Targuist (B)
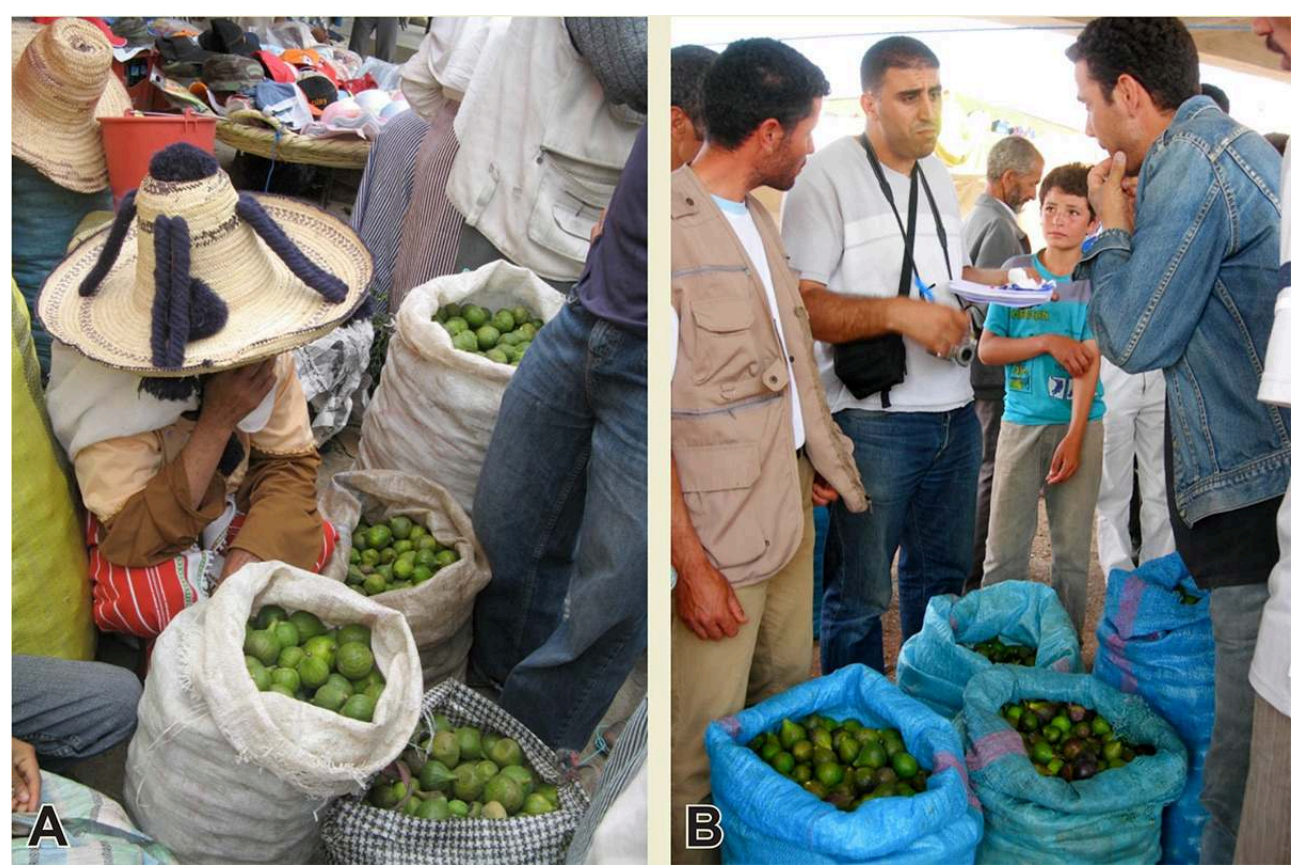

(c) Hmimsa Y

\section{Le figuier}

Dans la majorité du territoire rifain, le figuier est appelé šžər, ce qui signifie en arabe marocain « arbre ». Dans d'autres régions arabophones du Maroc, le figuier est nommé plutôt kərmūṣ ou karma qui veulent dire respectivement figue et figuier en arabe marocain. Dans le territoire amazighophone de notre aire d'étude, le mot tazart désigne indistinctement le fruit et l'arbre. Cependant šžar renvoie aux arbres de manière générale et à leur origine sylvestre.

Concernant sa répartition dans l'espace, le figuier est omniprésent, à côté des maisons, on trouve les variétés préférées pour la consommation familiale, généralement ce sont des variétés bifères, c'est-à-dire portant une production de figues fleurs parthénocarpiques, dénommée bākōr, au printemps et une production de figues d'automne parthénocarpique ou non-parthénocarpique dénommée kərmūṣ. Alors que dans les champs et les vergers, on trouve les autres types de variétés de figuier.

\section{Le figuier spontané}

21 Le figuier spontané est connu par les agriculteurs sous le nom vernaculaire de nābūt. À l'échelle du Rif, Il dispose d'un statut bien défini, en poussant tout seul, à partir d'une graine qui germe et donne soit un pied de figuier ou un caprifiguier. Du point de vue répartition spatiale du $n \bar{a} b \bar{u}$, et d'après les prospections que nous avons réalisé, nous avons constaté qu'il est lié à la présence de l'eau et fréquemment rencontré aux bords 
des oueds, des ruisseaux, canaux d'irrigation ou des puits (Figure 4). Cependant, et que ce soit à l'intérieur ou en bordure de champs, dès l'apparition de ces pieds, ils sont considérés comme un bien privé appartenant au propriétaire du puits ou de la parcelle : "Seul le propriétaire dispose de tout droit pour maintenir le pied spontané sur sa terre, le remplacer par un autre ou le greffer par une variété cultivée ${ }^{6}$.

Figure 4 : Quelques exemples de figuier spontané
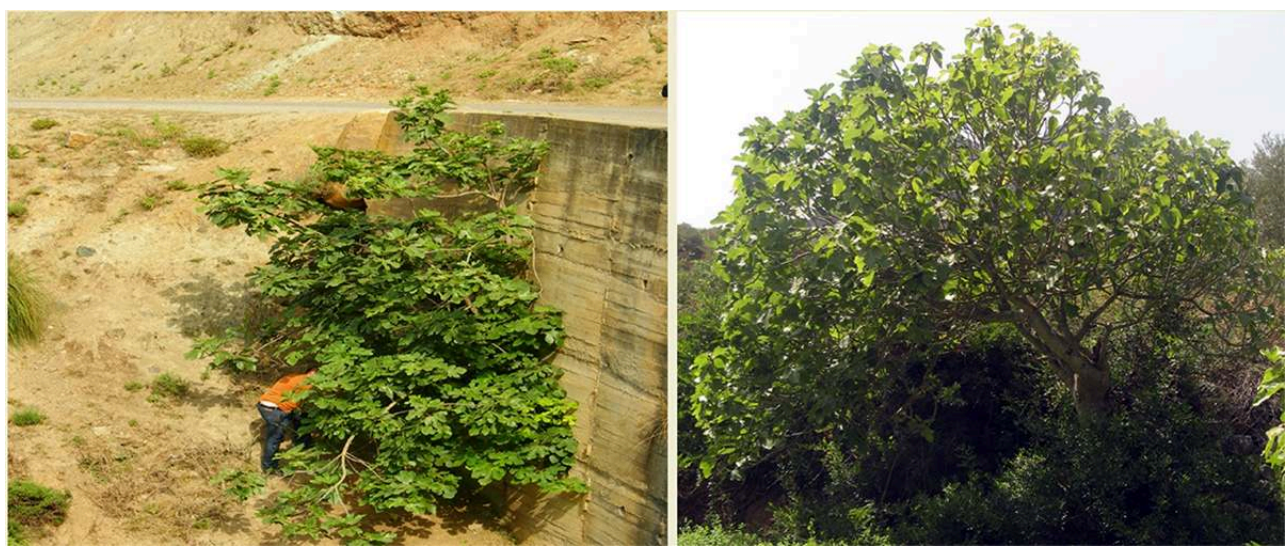

(c) Hmimsa Y.

Dans un autre contexte d'usage, ces figuiers spontanés lorsqu'ils sont situés sur des chemins ou dans des terrains non appropriés, ils sont sujets à une exploitation par les pauvres du village, essentiellement, les personnes qui ne disposent pas de figuiers. En plus, ces mêmes arbres, peuvent être greffés avec d'autres variétés de figuiers. La greffe peut se faire avec abnégation et d'une manière altruiste en greffant pour la gratitude envers Dieu fi sābil ḷ̣ăh. Cet acte s'inscrit, ainsi, dans une perspective d'offrir des figues comme offrande à Dieu : «On le donne dans le chemin de Dieu fi säbil l!̣āh, pour la satisfaction du Dieu, pour que le Dieu accepte nos bonnes actions $»^{7}$.

\section{Typologie, nomenclature et usages des formes spontanées}

La nature de ce type de figuier est difficile à déterminer dans l'immédiat. En effet, ce n'est que tardivement (généralement après trois ans) que les agriculteurs peuvent savoir s'il s'agit d'un figuier ou d'un caprifiguier. Selon les agriculteurs, la nature du fruit (figue) permet la distinction entre deux types de nābūt: le figuier spontané produisant des figues et le caprifiguier spontané produisant des caprifigues.

$\mathrm{Au}$ cours de nos travaux, nous avons constaté que le figuier spontané, est un figuier unifère (c'est-à-dire qu'il donne une seule production, les figues d'automne). D'autre part, ces figues sont souvent de mauvaise qualité et peu appréciée à cause de leur petite taille et un goût peu sucrée. En revanche, la diversité du figuier spontané est peu connue même si des variations de la taille et de la couleur des figues laissent penser qu'elle est importante. Dans certains cas, quand ces fruits s'avèrent bon, les paysans disent que le fruit est consommé. En plus, les paysans perçoivent des liens de filiation entre les figues spontanées et les figues cultivées à travers des caractères comme la couleur, la forme ou la date de maturité. Ainsi, lors de nos missions de prospection, nous avons rencontré des paysans qui appellent certaines variétés nābūt ġuddān. En 
demandant des explications, ils ont répondu que, malgré sa spontanéité, le fruit ressemble par sa forme, et sa couleur à celui de la variété guddān.

Lorsque le nābūt évolue en caprifiguier, ses caprifigues ne sont pas mangeables. En outre, ils sont utilisés dans la caprification quand les caprifiguiers font défauts. Cependant, les paysans évoquent le problème de la présence d'un parasite dans les caprifigues. Ils arrivent à distinguer le parasite du blastophage en affirmant que le parasite possède une queue rouge (Figure 5), mais ils le considèrent quand même comme un blastophage. Au niveau de la nomenclature vernaculaire, ils lui donnent en arabe le nom šonwīla et en berbère le nom tizit / tiziwt qui signifient respectivement " mouche / abeille». Ces noms sont utilisés également pour désigner le blastophage. Malgré cela, les différents types de figuiers spontanés sont conservés sur le terrain. Dans certains cas, nous avons noté des pratiques de greffage d'une variété de figuier cultivé ou d'une variété de caprifiguier sur les formes spontanées. Le greffage, de formes cultivées sur des formes spontanées, est l'un des pratiques bien ancrés dans le savoir local et pratiqué à grande échelle chez d'autres fruitiers comme l'olivier (Haouane 2012, Aumeeruddy-Thomas et al. 2016, Ater et al. 2016, Aumeeruddy-Thomas et al. 2017) ou la vigne (Hmimsa 2009, El Oualkadi et al. 2011).

Figure 5 : Identification de l'insecte parasite sur les caprifigues de nābūt

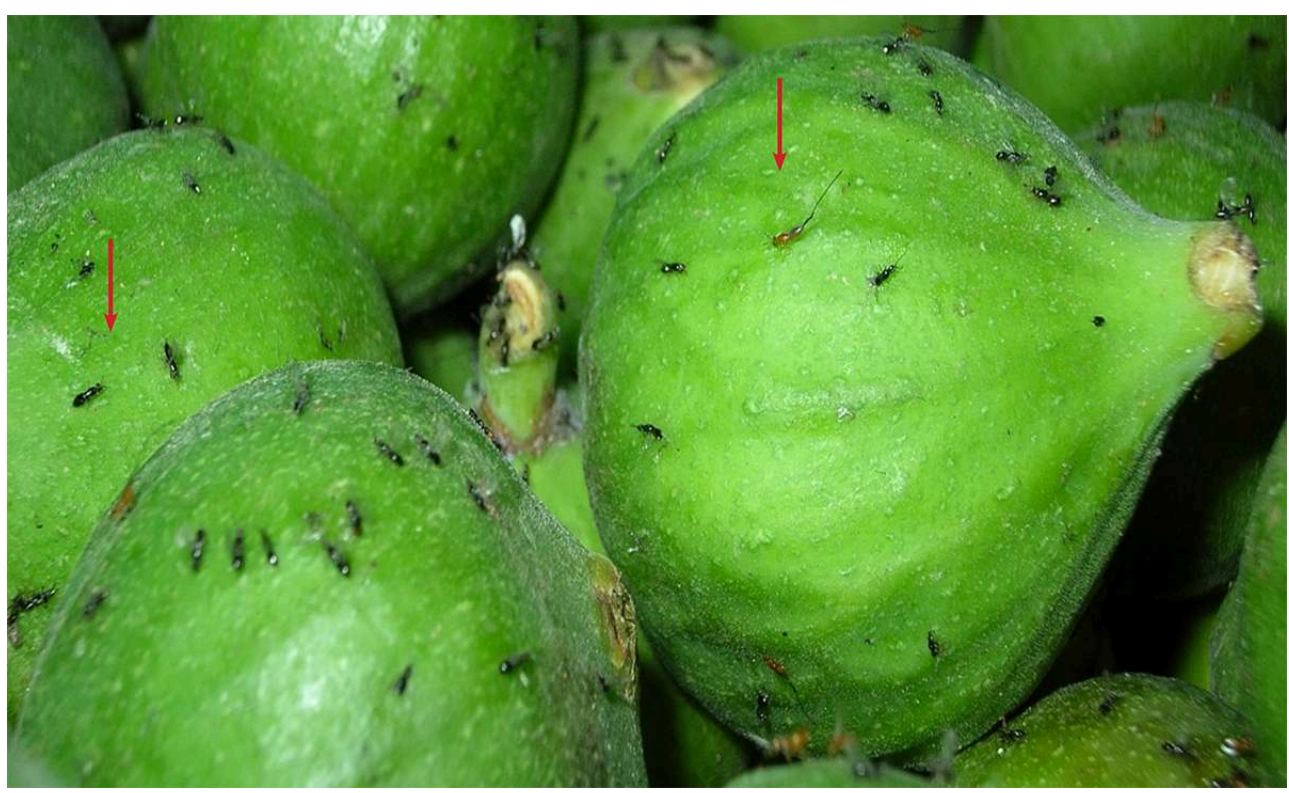

(c) Hmimsa Y.

Du point de vue de l'utilisation, nous avons constaté une variabilité dans les pratiques et les usages des figuiers et des caprifiguiers spontanés. Cependant, cette variabilité dépend d'un ensemble de facteurs, dont la disponibilité et la richesse en variétés de figuier et les préférences gustatives des gens. Ainsi, dans les villages où les figueraies sont peu développées, l'usage du caprifiguier spontané se limite à l'alimentation de bétail et les figues spontanées sont consommées. Alors que dans les villages où les figueraies sont développées, les pieds spontanés, qu'ils soient figuiers ou caprifiguiers, n'ont pas d'usages reconnus : "les nābūts de figuiers ne font pas de figues fleurs mais des figues de qualité médiocre et de goût piquant, alors que les nābūts dokkār font des caprifiques 
dont la šonwilla (blastophage) est dominé par une autre šanwïla avec une queue (pour désigner un parasite) détériorant les figuiers $»^{8}$.

\section{Statut du nābūt : spontané ou en cours de domestication?}

27 Au cours de nos travaux qui ont porté sur une large campagne de prospection qui a touché 198 villages au niveau des agroécosystèmes traditionnels du Rif, nous avons noté la reconnaissance du nābūt comme une variété de figuier dans seulement 6 dšar. En effet, (Figure 6), nous avons constaté dans ces dšar que le terme nābūt a un usage tout à fait différent de ce que nous avons présenté précédemment. Dans ces villages, le nābūt est une variété nommée locale de figuier que les agriculteurs sont capable de distinguer par rapport aux autres variétés. Selon ces agriculteurs, cette variété regroupe l'ensemble des pieds spontanés et sont classés comme une nouvelle variété s'ajoutant aux variétés existantes. En plus, elles bénéficient des même soins et pratiques (bouturage, taille...) que les variétés cultivées. Ces pieds spontanés se trouvent dans des villages caractérisés par des terres fertiles, l'abondance de l'eau et une importante diversité variétale du figuier (Figure 6). Les agriculteurs confirment que les pieds de figuiers regroupés sous le nom de nābūt ont poussé spontanément mais ils ont été sélectionnés et choisis pour la production de fruits comestibles. Il y a là un lien ténu entre compartiment spontané et compartiment cultivé du figuier, la façon de nommer démontrant le passage d'un arbre issu de semis à celui d'une variété cultivée. Il s'agit d'une dynamique de recrutement de nouvelles formes par les agriculteurs pour enrichir la diversité variétale du compartiment cultivé. On peut parler d'un processus de domestication continu et qui peut expliquer l'importance de la diversité variétale du figuier dans les agroécosystèmes rifains. Cette continuité linguistique a également été démontrée pour l'olivier dans la région du Rif où des noms de variétés d'oléastres se retrouvent chez des variétés cultivées présentant des caractéristiques morphologiques identiques aux caractères pré-sélectionnés chez les oléastres (Aumeeruddy-Thomas et al. 2017). Les études génétiques et interdisciplinaires réunissant ethnobiologie et génétique, corroborent et explicitent cette conception de la dynamique entre compartiments spontanés et cultivés en démontrant la proximité entre les deux types de génotypes et entre types nommés et spontanés (Khadari et al. 2009, Achtak et al. 2010, Aumeeruddy-Thomas et al. 2017). 
Figure 6 : Richesse variétale du figuier dans les six douars considérant le nābūt comme une variété cultivée

\section{Nombre de variétés}

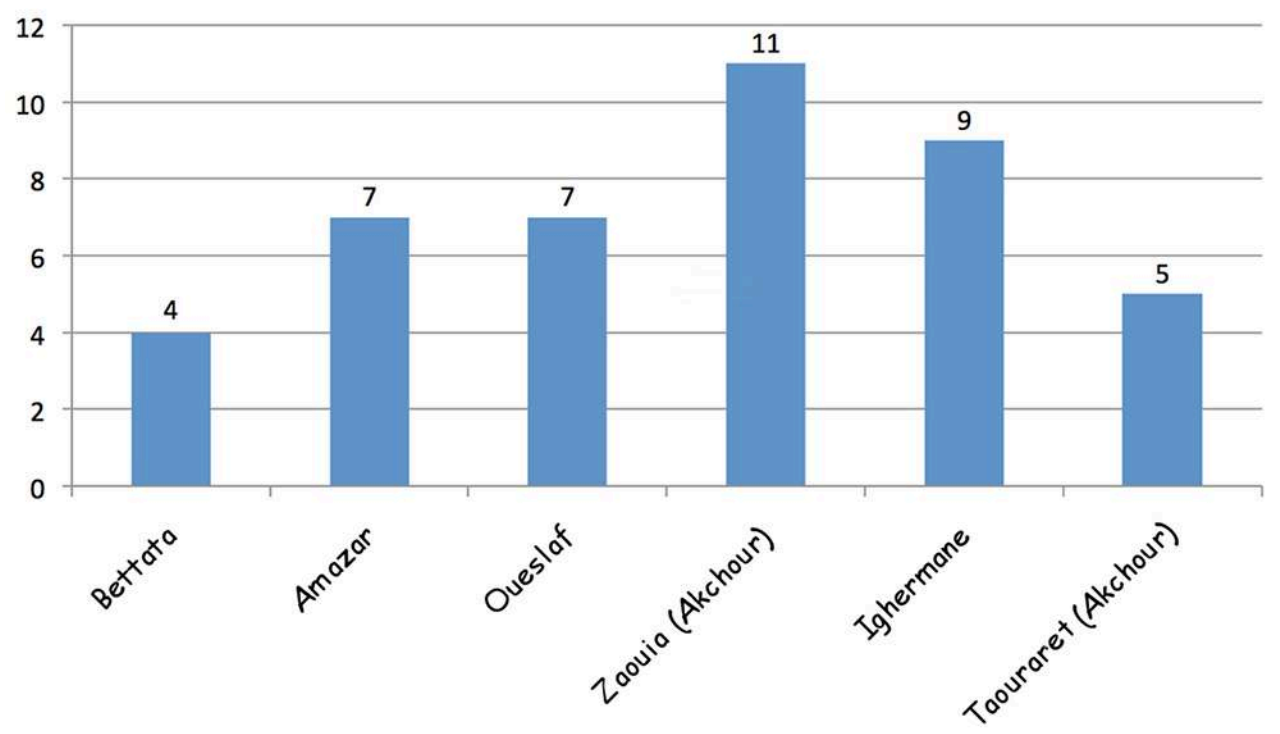

\section{Conclusion}

Le figuier est considéré comme représentatif de l'arboriculture fruitière dans les agroécosystèmes traditionnels du Rif, sans oublier sa valeur patrimoniale et sociale. D'une manière générale, différentes études ont souligné que la gestion de la diversité variétale par les agriculteurs est directement influencée par des facteurs sociaux, économiques, culturels et écologiques. Dans ce sens, nos travaux portant sur les liens cultivés - spontanés ont montré que lorsqu'il s'agit d'un figuier ou d'un caprifiguier, le spontané présente une des caractéristiques de transmission et d'héritage identique. Il a poussé sans intervention humaine, mais cela ne l'empêche pas de devenir un bien arboricole comme les autres faisant de ce spontané un produit social et non naturel. Son lien profond au domaine du sacré, rajoute en outre une dimension culturelle forte à ce spontané, car fondé sur des croyances qui relie les hommes à leur territoires, par le biais de la bénédiction divine ou baraka. L'agriculteur peut maintenir la variété spontanée quand elle donne un fruit de bonne qualité, la greffer ou l'éliminer. Un acte qu'on peut inscrire comme étant la base d'une pratique qui fait partie du processus de domestication par recrutement de nouveaux morphotypes qui par le biais des croisements et de la multiplication sexuée présentent un nouveau génotype. Le statut de spontané peut changer pour évoluer vers le cultivé suite à l'intérêt manifesté par l'agriculteur et les pratiques qu'il exercera sur le spontané. En effet, dès que le gens commencent à le consommer, à lui accorder de l'intérêt et du temps pour le tailler, le bouturer et le replanter, il peut être considéré comme une variété à part et les agriculteurs le citent parmi les variétés de figuier qu'ils ont dans leurs vergers. Sans doute, pourrions-nous parler aussi d'une réappropriation d'échappées de culture nābūt qui correspond au processus de sélection qui a pu au cours du temps représenter une approche de domestication du figuier. 


\section{BIBLIOGRAPHIE}

Achtak H., Ater M., Oukabli A., Santoni S., Kjellberg F. \& Khadari B. 2010 - Traditional agroecosystems as conservatories and incubators of cultivated plant varietal diversity : the case of fig (Ficus carica L.) in Morocco. BMC Plant Biology $10: 28$

Ait Kaki M. 2003 - Les Etats du Maghreb face aux revendications berbères. Politique étrangère 68 (1) : 103-118.

Akpavi S., Chango A., Tozo K., Amouzou K. \& Akpagana K. 2013 - Valeur nutrition/santé de quelques espèces de Légumineuses alimentaires mineures au Togo. Acta botanica Gallica: bulletin de la Société botanique de France 155 (3) : 403-414

Ater M., Essalouh L., Ilbert H., Moukhli A. \& Khadari B. (Ed.) 2016 - L'oléiculture au Maroc de la préhistoire à nos jours : pratiques, diversité, adaptation, usages, commerce et politiques. Options Méditerranéennes : Série A (Séminaires Méditerranéens ; n. 118) Montpellier : CIHEAM. 215 p. [En ligne] http://om.ciheam.org/om/pdf/a118/a118.pdf [consulté le: 24/06/2016].

Aumeeruddy-Thomas Y. 2010 - Des clones aux semis : domestication des arbres en Méditerranée, un continuum entre nature et culture. Exemple de Ficus carica L., Olea europaea L. et Castanea sativa L.. In Delhon C., Théry-Parisot I. \& Thiébault S. (dir.) Des Hommes et des plantes : Exploitation du milieu et gestion des ressources végétales de la préhistoire d'Antibes. APDCA : 379-390

Aumeeruddy-Thomas Y., Hmimsa Y., Ater M. \& Khadari B. 2014 - Beyond the divide between wild and domesticated: spatiality, domesticity and practices pertaining to fig (Ficus carica L.) and olive (Olea europaea L.) agroecosystems in Morocco. In : Chevalier A., Marinova E. \& Peña-Chocarro L. (Ed.) Crops and people: choices and diversity through time. Brussels, Earth EU, London, OXFAM : 191-197.

Aumeerudddy-Thomas Y., Bailly A., Alleaume S. \& Hmimsa Y. 2016 - Grafted oleaster-olive agrosylvopastoral systems in Northern Morocco. In Thiébault S. \& Moatti J-P. (Ed.) The Mediterranean Region Under Climate Change. A Scientific Update. IRD Editions, Marseille : 523-532. [En ligne] http://fr.calameo.com/read/00331938471440c995c73 [Consulté le 5/2/2017].

Aumeeruddy-Thomas Y., Moukhli A., Haouane H. \& Khadari B. 2017 - On going domestication and diversification in grafted olive-oleaster agroecosystems in Northern Morocco. Reg Environ Change $17: 1315-1328$

Benabid A. 1982 - Étude phytoécologique, biogéographique et dynamique des associations et séries sylvatiques du Rif occidental. Thèse de Doctorat, Université d'Aix Marseille, $199 \mathrm{p}$

Bourguiba H., Audergon J.M., Krichen L. Trifi-Farah L., Mamouni A., Trabelsi S. \& Khadari B. 2012 - Genetic diversity and differentiation of grafted and seed propagated apricot (Prunus armeniaca L.) in the Maghreb region. Scientia Horticulturae 142: 7-13.

Camps G. 1983 - Comment la Berbérie est devenue le Maghreb arabe. Revue de l'Occident musulman et de la Méditerranée (Aix-en-Provence) 35 : 7-24.

Castañeda-Álvarez N.P., Khoury C.K., Achicanoy H.A., Bernau V., Dempewolf H., Eastwood R.J., Guarino L., Harker R.H., Andy Jarvis A., Maxted N., Müller J.V., Ramirez- Villegas J., Sosa C.C., Struik P.C., Vincent H. \& Toll J. 2016 - Global conservation priorities for crop wild relatives. Nature plants 16022 | DOI: 10.1038 / NPLANTS. 2016. 22 
Corriente F., Pereira Ch. \& Vicente Á (Ed.) 2017 - Encyclopédie linguistique d'Al-Andalus Vol. 2 : Dictionnaire du faisceau dialectal arabe andalou. Perspectives phraséologiques et étymologiques. Berlin/ Boston, De Gruyter, 1238 p.

Delplancke M. 2011 - Histoire évolutive de l'amandier cultivé (Prunus dulcis) en Méditerranée: regards croisés sur la domestication, dialogue entre la biologie et l'ethnobiologie. Thèse en Biologie des populations, Université Montpellier 2, Montpellier (France).

El Oualkadi A., Ater M., Messaoudi Z., El Heit K., Laucou V., Boursiquot J.M., Lacombe T. \& This P. 2011 - Genetic diversity of Moroccan grape accessions conserved ex situ compared to Maghreb and European gene pools. Tree Genetics \& Genomes. DOI : 10.1007/s11295-011-0413-3

Fay G. 1972 - Recherche sur l'organisation de la vie rurale et sur les conditions de la production dans la basse montagne rifaine, chapitre 3 : Olivier. Thèse de troisième cycle, Université de Paris VII : 393-442 Focauld C. de 1998 (1883-1884) - Reconnaissance au Maroc. Paris, L'Harmattan, 240 p.

Galluzzi G., Eyzaguirre P. \& Negri V. 2010 - Home gardens: neglected hotspots of agrobiodiversity and cultural diversity. Biodiversity Conservation DOI 10.1007/s10531-010-9919-5

Haouane H. 2012 - Origines, domestication et diversification variétale chez l'olivier (Olea europaea L.) à l'ouest de la Méditerranée. Thèse de doctorat, Marrakech, Université Cadi Ayyad, Faculté des Sciences Semlalia, $272 \mathrm{p}$.

Hmimsa Y. 2009 - L'agrodiversité de l'agroécosystème à l'arbre : cas du Rif (Nord du Maroc). Thèse de doctorat. Tétouan, Université Abdelmalek Essaadi - Faculté des Sciences. $328 \mathrm{p}$

Hmimsa Y., Aumeruddy-Thomas Y. \& Ater M. 2012 - Vernacular Taxonomy, Classification and Varietal Diversity of fig (Ficus carica L.) Among Jbala cultivators in Northern Morocco. Human Ecology 40 : 301-313.

Hmimsa Y., Aumeeruddy-Thomas Y. \& Ater M. 2017 - Lexique sur les figuiers: Exemple des variétés nommées chez les Beni Ahmed (Jbala, Rif occidental). In : Vicente Á., Caubet D. \& NaciriAzzouz A. (Ed.) La Région du Nord-Ouest marocain : parlers et pratiques sociales et culturelles. Prensas de la Universidad de Zaragoza : 265-274.

INRA 2011 - Atlas du palmier dattier au Maroc. Rabat, 197 p.

Iraqui Sinaceur Z. (Ed.) 1993 - Le dictionnaire Colin d'arabe dialectal marocain (arabe français). Rabat, Al Manahil, 1877 p.

Jean Léon l'Africain (El Hasan ben Mohammed el-Wazzan ez-Zayyâti) 1981 (1550) - Description de l'Afrique. 2 Vol., 630 p. Nouvelle édition traduite de l'italien par A. Epaulard et annotée par A. Epaulard, Th. Monod, H. Lhote et R. Mauny. Paris

Khadari B., Grout C., Santoni S. \& Kjellberg F. 2005 - Contrasted genetic diversity and differentiation among Mediterranean populations of Ficus carica L.: a study using mtDNA RFLP. Genetic resources and crop evolution : 97-109.

Khadari B., Achtak H. \& Kjelllberg F. 2009 - Tracking the genetic signature to identify fig origins: Insights for evolution before and during domestication. Actes: The Fourth International Symposium on fig. Meknes, Morocco.

Laouina 1998 - L'environnement des montagnes du Nord : Atouts, contraintes et processus de dégradation. In : Berriane M. \& Laouina A. Le Développement du Maroc Septentriona : 15-59.

Larousse 2017 - [En ligne] http://www.larousse.fr/dictionnaires/francais [consulté le 26/06/2017)]. 
Lery F. 1982 - L'agriculture au Maghreb ou pour une agronomie méditerranéenne. Paris, Maisonneuve et Larose, $337 \mathrm{p}$.

Łuczaj L., Pieroni A., Tardío J., Pardo-de-Santayana M., Sõukand R., Svanberg I. \& Kalle R. 2012 Wild food plant use in 21st century Europe: the disappearance of old traditions and the search for new cuisines involving wild edibles. Acta Soc Bot Pol 81 (4) : 359-370.

Lulekal E., Asfaw Z., Kelbessa E. \& Van Damme P. 2011 - Wild edible plants in Ethiopia: a review on their potential to combat food insecurity. Afrika focus 24 (2) : 71-121.

Mamouni A., El Bakkali A., Lambert P., Krichen L., Ahmed Oukabli A., Audergon H.M., Chatelet P., El Modafar C. \& Khadari B. 2013 - Bottleneck and gene flow effects impact the genetic structure of seed-propagated apricot populations in Moroccan oasis agroecosystems. Plant Genetic Resources: Characterization and Utilization : 1-11 doi:10.1017/S1479262113000543

Maurer G. 1968 - Les paysans du Haut Rif central. Revue de Géographie du Maroc 14 : 3-70.

McKey D., Elias M., Pujol B., Duputié A., Delêtre M. \& Renard D. 2012 - Maintien du potentiel adaptatif chez les plantes domestiquées à propagation clonale. Leçons de gestion par les cultivateurs de manioc amérindiens. Revue d'ethnoécologie 1.

Mimoun A. 2003 - La sociedad rifeña frente al Protectorado español de Marruecos (1912-1956). Barcelona, Bellatera, $270 \mathrm{p}$.

Moscoso F. 2003 - El dialecto árabe de Chauen (N. de Marruecos) Estudio lingüístico y textos. Cádiz, Universidad de Cádiz, 382 p.

Niveditha T.M.A. 2017 - Wild edible plants of India - A review. International Journal of Academic Research. 4 (3) (1) : 189-198.

Oukabli A. 2003 - Le figuier: un patrimoine génétique diversifié à exploiter. Bulletin mensuel d'information et de liaison du PNTTA 106, 4 p.

Sadiki M., Birouk A., Bouizgaren A., Belqadi L., Rh'rib K., Taghouti M., Kerfal S., Labhilili M., Bouhya H., Douiden R., Saidi S. \& Jarvis D. 2002 - La diversité génétique in situ du blé dur, de l'orge, de la luzerne et de la fève : options de stratégie pour sa conservation. In : Birouk A., Sadiki M., Nassif F., Saidi S., Mellas H., Bammoun A. \& Jarvis D. (Ed.) La conservation in situ de la biodiversité agricole: un défi pour une agriculture durable. IPGRI : 37-117.

Simkova K. \& Polesny Z. 2015 - Ethnobotanical review of wild edible plants used in the Czech Republic. Journal of Applied Botany and Food Quality 88 : 49-67, DOI:10.5073/JABFQ.2015.088.009

Sonnini C.S., Veillard E. \& Chevalier E. 1810 - Vocabulaire portatif d'agriculture d'économie rurale et domestique, de médecine de l'Homme et des animaux, de botanique, de chimie, de chasse, de pêche, et autres sciences ou arts qui ont rapport à la culture des terres et à l'économie. Buisson F., 485 p.

Soriano Niebla, Juan J. (Ed.) 2004 - Hortelanos de la Sierra de Cádiz. Las variedades locales y el conocimiento campesino sobre el manejo de los recursos genéticos. Mancomunidad de municipios de la Sierra de Cádiz. Villamartín, Cádiz. 240 p

Tardío J., Pardo-De-Santayana M. \& Morales R. 2006 - Ethnobotanical review of wild edible plants in Spain. Botanical Journal of the Linnean Society $152: 27-71$.

Pardo-de-Santayana M., Tardío J., Blanco E., Carvalho A.M., Lastra J.J., Miguel E.S. \& Morales R. 2007 - Traditional knowledge of wild edible plants used in the northwest of the Iberian Peninsula (Spain and Portugal): a comparative study. Journal of Ethnobiology and Ethnomedicine $3: 27$ doi: 10.1186/1746-4269-3-27 
Tavaud M., Zanetto A., David J.L., Laigret F. \& Dirlewanger E. 2004 - Genetic relationships between diploid and allotetraploid cherry species (Prunus avium x Prunus gondouinii and Prunus cerasus). Heredity $93:$ 631-638.

Tayou A. 1985 - Étude technique et économique de la culture du figuier dans la région de Chefchaouen. Mémoire d'Ingénieur Horticole. Institut Agronomique et Vétérinaire Hassan II (IAV). Rabat, Maroc, $100 \mathrm{p}$.

Teniente C.C. 1928 - Geografía de Gomara. Alta Comisaria de Espana en Marruecos : 7-26.

Vicente Á., Caubet D. \& Naciri-Azzouz A. (Ed.) 2017 - La région du Nord-Ouest marocain: Parlers et pratiques sociales et culturelles. Zaragoza, Prensas de la Universidad de Zaragoza, $362 \mathrm{p}$.

Walali Loudiyi D. 2002 - Quelques espèces fruitières d'intérêt secondaire cultivées au Maroc. In : Llácer G., Aksoy U. \& Mars M. (Ed.) Cultures fruitières sous-utilisées dans la région méditerranéenne. CIHEAM-IAMZ (Zaragoza) : 47-62. [En ligne] http://ressources.ciheam.org/om/pdf/ c13/96605640.pdf [consulté le 13/06/2017]

\section{NOTES}

1. Il faut noter que le caroubier est considéré comme un fruitier domestiqué. Au Maroc, il reste une essence forestière naturelle non domestiquée et il n'existe ni variétés reconnues ni une tradition de planter des vergers. Les arbres exploités et parfois greffés sont toujours spontanés.

2. Il s'agit de forme échappée de culture par semis et qui se propage par la dissémination des fruits et graines des formes cultivées qui se multiplient exclusivement par voie végétative.

3. Hameau ou regroupement d'habitations et d'individus dans la montagne.

4. Le terme Caïd ( qāid) peut faire référence à un représentant de l'autorité locale au niveau du dšar, alors que ce même nom a été attribué avant aux notables qui rassemblaient avant entre le pouvoir et les fonctions administratives, financières et judiciaires.

5. Ce marché se trouve dans une zone de rencontre entre l'arabe et l'amazigh, dans ce sens, l'ensemble des noms vernaculaires que nous avons recueillis se caractérisent par la présence d'un préfixe « $a$ ? » qui démontre la berbérisation de ces termes (Hmimsa et al. 2012).

6. Dire d'un agriculteur interviewé à Talandaoued (Bni Ahmed)

7. Idem 6

8. Idem 6

\section{RÉSUMÉS}

Le figuier est un arbre fruitier emblématique de la Méditerranée au même titre que l'olivier, la vigne ou l'amandier. Sa culture est très répandue dans les montagnes du Rif où elle représente un élément caractéristique des agroécosystèmes traditionnels. La diversité variétale est exceptionnelle, elle est estimée à plus de 100 variétés locales. D'un point de vue socioéconomique, le figuier est très apprécié par les populations locales. En effet, au-delà de son importance alimentaire et économique, le figuier a une valeur patrimoniale importante. L'approche ethnobiologique a permis d'apporter des éléments d'explication aux différentes questions liées aux processus sociaux conduisant à la sélection et à la conservation de cette 
diversité variétale.

Dans le cadre de cette diversité on relève la coexistence de formes cultivées (variétés nommées) et spontanées ( $n \bar{a} b \bar{u} t)$ dans les vergers traditionnels. Ce qui nous amène à nous interroger sur l'origine et la conservation de la diversité chez le figuier en relation avec la présence des formes spontanées qui se multiplient par voie sexuée par opposition au figuier cultivé qui est multiplié par les hommes par boutures. En effet, nābūt, arbre type du figuier spontané, présente un statut controversé en fonction des zones, des usages et des pratiques. Ces aspects feront l'objet de notre communication.

The fig tree is an emblematic fruit tree of the Mediterranean region as much as olive, grapevine or almond. Its cultivation is widespread in the Rif Mountains where it has a characteristic feature of traditional agroecosystems. Varietal diversity is exceptionally high, estimated at over 100 local varieties. From a socio-economic perspective, the fig tree is highly appreciated by the local populations. Indeed, beyond its food and economic importance, the fig tree has an important heritage value. The ethnobiological approach has provided explanatory elements to the various issues related to the social processes leading to the selection and conservation of this varietal diversity.

Within this diversity, the coexistence of cultivated (named varieties) and spontaneous (nābūt) forms in traditional orchards is noted. This leads us to explore the origin and conservation of the diversity of the fig tree in relation to the presence of spontaneous forms. Indeed, nābūt, typical variety of the spontaneous fig tree which propagates sexually though seedlings as opposed to cultivated varieties - which are propagated clonally by men through cuttings - is controversial depending on the zones, the uses and the practices. These aspects will be the subject of our communication.

\section{INDEX}

Mots-clés : Rif, figuier, agroécosystèmes traditionnels, spontané, cultivé, usage, pratiques

Keywords : Rif, Fig tree, traditional agroecosystems, spontaneous, cultivated, use, practices

\section{AUTEURS}

\section{YOUNES HMIMSA}

Enseignant chercheur à l'Université Abdelmalek Essaâdi, Département des Sciences de la Vie (Biologiste et ethnobotaniste, Membre du laboratoire de Botanique appliquée (Équipe BioAgrodiversité)), Faculté Polydisciplinaire de Larache. B.P. 745, Poste Principale, Larache 92004 MAROC.

hmimsayounes@gmail.com

\section{YILDIZ AUMEERUDDY-THOMAS}

Directeur de Recherche, CEFE-CNRS, UMR 5175, Responsable Équipe Interactions Bioculturelles. 1919, route de Mende F-34293 Montpellier Cedex 5

yildiz.aumeeruddy-thomas@cefe.cnrs.fr

\section{MOHAMMED ATER}

Professeur de l'enseignement supérieur à l'Université Abdelmalek Essaâdi, Département de Biologie (Biologiste et Écologue, Membre du laboratoire de Botanique appliquée (Équipe Bio- 
Agrodiversité)), Faculté des Sciences, PB. 2062, Tétouan, 93030, MAROC.

Mohammed.ater@gmail.com 